\title{
Pop culture and social insertion: How can play in adolescence and adulthood be "therapeutic"?
}

\author{
Anne M. Goodall* and Alexis H. Truong*
}

\begin{abstract}
In this study we explore how participation in tabletop role-playing games (TTRPGs) fosters experiences of social insertion in adolescence and adulthood. We conducted semi-directed interviews with nine participants who identified themselves as having used such practices to overcome difficult and challenging life experiences. We look at how participants interpreted their play experiences, described by some as "therapeutic." Through TTRPGs, players were able to explore and better understand aspects of themselves, explore new interactions, and "test" new ways of expressing themselves. Additionally, participants were able to develop their interpersonal skills by participating in such practices, namely because of the roleplaying element. Participants stated that these practices and their therapeutic qualities also had positive effects on their lives outside of the game, helping them to enter and engage in various social situations that they previously felt excluded from, or hesitant to participate in. We argue that social interventions could invest in these types of cultural practices, embedded in popular culture, to encourage and facilitate participation of adolescents and adults in mental health services.
\end{abstract}

Key Words Mental health; role-playing; well-being; social insertion; marginalisation.

\section{INTRODUCTION}

How do tabletop role-playing games (TTRPGs) help prevent experiences of social exclusion in youths and young adults? In the late 1970s and in the 1980s, TTRPGs were popularized through the publication of games like Dungeons and Dragons (D\&D) (Bowman, 2010). Role-playing games (RPGs) generally require the players to adopt the role of a character, interact with others through this character and experience a collectively imagined and sustained world for the purpose of the game. Objectives often focus on completing quests and adventures, though some games also focus on storytelling and world exploration. Examples of non tabletop RPGs include video games, such as The Legend of Zelda series, and live action role-playing games (LARPs) like Vampire: The Masquerade. Video games are played on virtual platforms with an animated version of a character, while LARPs generally involve elaborate costumes and physically acting out characters. On the other hand, TTRPGs like D\&D resemble more traditional board games, as they are mostly played with no more than twelve players, and require only a pen and paper, a rule book, and a set of dice (Wyatt et al., 2014). In TTRPGs, no tangible representations of the characters or the environment is necessary: game play occurs nearly entirely within the players' imagination, the pen and paper are used to keep track of the characters' statistics and possessions, and dice are used to determine the success of actions. Moreover, some individuals and groups involved in the TTRPG community propose that the main objective is not winning or losing, but rather appreciating a collective experience of creating and inhabiting this immersive fictional realm (Schiesel, 2008).

In the early days of the 1980 moral panic that surrounded TTRPGs like D\&D (Martin \& Fine, 1991), these practices were framed as cult-like communities that targeted socially excluded individuals or further fanned retreat from the "real world" and social anomy (Martin \& Fine, 1991). However, recent scholarship has shown that similar practices can actually foster social inclusion and social and professional insertion (Truong \& Gaudet, 2020), much like other forms of leisure (Rowe, 2014; Smallfield \& Molitor, 2018). Although little has been written on the topic, contrary to popular belief, such role-playing practices could actually help address social exclusion (Truong, 2013). The term social exclusion is commonly associated with bullying, but social exclusion can occur due to a variety of reasons (Lynn Mulvey, Boswell \&

Correspondence to: Anne M. Goodall, Department of Criminology, Faculty of Social Sciences, University of Ottawa, 1-97 Ste-Cecile St, Ottawa, ON K1L 5L4, Canada. E-mail: agood019@vottawa.ca

To cite: Goodall, A. M., \& Truong, A. H. (2021). Pop culture and social insertion: How can play in adolescence and adulthood be "therapeutic"? Journal of Community Safety and Well-Being, 6(1), 17-21. https://doi.org/10.35502/jcswb.178

(C) Author(s) 2021. Open Access. This work is distributed under the Creative Commons BY-NC-ND license. For commercial re-use, please contact sales@sgpublishing.ca.

g.PUBLISHING Published by SG Publishing Inc. CSKA Official publication of the Community Safety Knowledge Alliance. 
Zheng, 2017). As a widespread issue gaining recognition, the United Nations defines social exclusion as "a state in which individuals are unable to participate fully in economic, social, political and cultural life, as well as the process leading to and sustaining such a state" (United Nations, 2016, p. 18). Social exclusion is also considered a risk factor for decreased prosocial behaviour, low self-esteem and mental health struggles, and even criminal activity (Coyne et al., 2011; Rowe, 2014; Stanley \& Arora, 1998).

In this text, we argue that the playfulness that characterizes these practices deters normative pressures associated with interactions in a way that also fosters social participation and inclusivity. We believe such practices could provide clues to innovate in the field of social intervention, especially in regard to young marginalized populations, by investing in their shared interests and cultural practices. We start by providing a bit of context and by introducing our methodology, and then move to present some of our data and interpretations.

\section{OUTCOMES OF LEISURE}

Tabletop role-playing games emerged in the 1970s and grew in popularity as they spread through high schools and colleges in North America (Kushner, 2017). However, they quickly became labelled by some as being associated with satanism, murder, suicide, and crime (Bowman, 2010; Martin \& Fine, 1991). An advocacy group called "Bothered About Dungeons \& Dragons" (BADD) claimed that D\&D was corrupting young minds, leading them to commit murders and suicide (Dempsey et al., n.d.). Mental health-related issues were seen as an increased risk factor, leading children with late-schizophrenia towards bad moral behaviour or causing depression in children because of the game's high emotional involvement (Dempsey et al., n.d). The media also helped to spread this image of TTRPGs. Most notably, an episode of the popular investigative news program 60 Minutes heavily criticized the game, arguing that children can become obsessed with the game, blurring reality and fiction in a way that could encourage some criminal behaviour (CBS, 1985). This negative media attention caused the game to be banned in many schools and youth programs, often due to protesting parents (Martin \& Fine, 1991). Today, the stigma against these practices has diminished greatly, as "geek" and mainstream culture become more and more intertwined (Cohen, 2014). Tabletop role-playing games have become more popular, as is made evident by television shows like The Big Bang Theory, movies like Disney's Onward, and a surge of popular podcasts on the topic, as well as with schools creating D\&D clubs for children not interested in sports, and restaurants and bars creating gaming nights for adults interested in this pastime (Barnes, 2018; Jahromi, 2017; Kraus, 2018).

While leisure is not considered essential to life, it has been recognized as important since Antiquity (Rudnik, 2005) and has been shown to have positive effects on health, self-esteem, and quality of life (Chen \& Chippendale, 2018; Rudnick, 2005). It has also been found to satisfy an individual's psychological needs, which in turn can have a positive effect on physical health, mental health, life satisfaction, and personal growth (Caltabiano, 1995; Chen \& Chippendale, 2018). Leisure activities have also been used as a form of social intervention to assist individuals who experience social isolation by providing them with a social network and opportunities to participate socially (Smallfield \& Molitor, 2018). For example, programs like Project Friendship and The Citizenship Project use leisure activities to help integrate marginalized individuals into the community (Rowe, 2014). Such networks can provide individuals with the opportunity to discuss their problems, share their experiences and receive emotional support (Caltabiano, 1995).

\section{METHODOLOGY}

We conducted semi-directed interviews with nine participants between June 2019 and January 2020. We decided to invest in longer, more in-depth interviews with a smaller number of participants in order to pay close attention to their lived experience and to the place and role of these practices in their lives. Because of this, statistical generalization of results is not possible. Interviews were conducted with participants who self-identified as having overcome hardships through their participation in TTRPGs. They were also chosen based on the extent, distinctiveness, and diversity of their experiences in these practices. Participants were aged between 23 and 56 years old, with experiences in TTRPGs ranging between 1 and 44 years in length. Six participants identified as male and three participants identified as female-with one participant also identifying as transgender male and one also identifying as transgender female during the interview.

During interviews, participants were asked to describe how TTRPGs helped them overcome challenging or difficult life experiences. Interviews were organized around three main themes: describing their most marking TTRPG experiences; describing the difficult or challenging life experiences they had overcome through their participation in TTRPGs, and describing the place and the role of TTRPGs in their lives since their first encounters with these practices. Interviews were recorded with an audio-recorder and subsequently transcribed. We used the qualitative data analysis software QDA Miner (Provalis Research) to code and analyze transcripts. We started with a "vertical" analysis of each interview, coding emerging themes and identifying the "story" shared by participants as well as variations and recurring elements. We then moved to doing a "horizontal" analysis throughout the various interviews in order to compare and integrate the various themes (see Gaudet \& Robert, 2018, for a more detailed description of this approach). This allowed us to classify the original 52 nodes identified inductively in our analysis into a series of primary, secondary, and tertiary categories of concepts. One of the main themes that emerged is this notion of "therapeutic experiences" shared by participants.

\section{THERAPEUTIC QUALITIES OF TTRPGS}

On numerous occasions, participants either expressed or evoked the idea that TTRPGs had a "therapeutic effect." Three participants explicitly described these practices as being "therapeutic," while the other participants spoke of related notions working towards well-being, which we consider a common thread for varying forms of therapeutic care (e.g., Binfet, 2017; Brooker \& Duce, 2000; Sweeney, 2009). 
Few authors have explored this topic in relation to such play practices. Blackmon (1994) described a specific case where D\&D had been used in therapy to help a player explore their personal identity. Others have explored the use of TTRPGs in social rehabilitation, helping individuals to develop skills and behaviours to navigate social situations (Rosselet \& Stauffer, 2013; Zayas \& Lewis, 1986). However, we still know little about why these practices seem especially relevant for participants in terms of healing or overcoming life difficulties. As Ashley, a participant, put it:

[TTRPGs] allow me to work through feelings of rejection, or pick an issue. Social exclusion, anything, questions around not having money, all of these sort of things, which we're able to do in a cooler setting. [...] Outside of games, the only real place that I have to work through some of the stuff that I'm working through with some of my characters is therapy, and that is an hour a week as opposed to three hours.

Playing a character that she identified with provided her the opportunity to work through some challenges or difficulties she had experienced, outside the conventional space of her formal therapy sessions. Ashley also expressed that playing such characters helped her reflect on and identify other issues she was facing, and facilitated raising them to her therapist. Even though participants did express playing these games as ludic activities, it appears that they facilitated a type of introspection conducive to a certain sense of well-being.

Expanding on this idea, participants also described how play allowed them to better understand certain aspects of who they are, were, or could become. Tabletop role-playing games were characterized as open worlds that allowed seemingly infinite possibilities for character creation and play. Participants explained that this gave them "agency" to explore various ways of being. Adam described how roleplaying was an opportunity to distance himself from his own behaviours and be critical of certain aspects of himself he felt were more "negative":

Being able to separate out that part of myself, and putting it in a character, and going "oh, that guy's an asshole." It's much easier than saying "oh, I'm a bad person." It's taking a behaviour and saying, "oh, that's bad behaviour". I don't have to... It's just behaviour. Behaviour changes.

Artistic forms of therapy like dance and drama therapy have been shown to develop an individual's self-esteem, identity, and confidence in skills as well as their motivation to change (Heard et al., 2013). Here, by gaining some distance, Adam felt it became possible for him to conceive personal change that did not feel threatening but, rather, was an opportunity for personal growth. This distance also allowed participants to explore and discuss various complicated and socially relevant notions such as sexism, racism, and corruption-on their own terms.

Identifying with characters was especially important in facilitating therapeutic effects experienced by participants. Many participants explained how character creation allowed them to role-play different characters that always reflected, to some degree, aspects of themselves. One way that they did this was by creating characters who represented "idealized" versions of themselves, allowing them to "practice" being and acting differently. A common example that was cited was the use of characters who were particularly "outgoing" and "socially adept," which allowed participants who otherwise described themselves as "introverted" or "socially awkward" to perform a confident self-presentation. Furthermore, much like the previous example shared by Adam, participants also used the freedom associated with character creation to generate characters who had lived similar experiences, or shared more negative or less desirable qualities with the players. This, in turn, helped them reflect and learn about themselves, the world around them, and what they felt to be their place in this world.

Participants also spoke of their TTRPG experiences as being "cathartic." They said that this kind of play opened an "inclusive" space and allowed them to express and release their emotions without the fear of consequences outside of play. This was the case, for example, when participants felt angry or stressed, but also felt like they did not have the means to express said emotions outside of play. As David stated: "RPGs are good for just dealing with that frustration. [If you are playing a fighter,] just the fact that you're pounding on something [...] [that's] enough to help you deal with some of the [...] frustrations that happen in everyday life." James explained that playing such characters allowed him to "feel powerful," a sense of power that he did not often feel outside of play, having experienced a lot of bullying. Role-playing was therefore a way to channel intense emotions that were explained as "difficult to deal with" in everyday life, but also a means of experiencing positive emotions that he rarely encountered outside of play. Like in other creatively geared therapeutic programs, such as dance and drama therapy, these opportunities may help individuals explore their own emotions and learn to better express their feelings (Frigon, 2010; Heard et al., 2013).

Similarly, for some participants, TTRPGs gave them an opportunity to express facets of their identity that were either unrecognized outside of their play community, perceived as less valued by others, or outright stigmatized. Both participants who identified as transgender reported using the game as an opportunity to express and explore how they defined themselves in a way that they felt they could not outside of the game. For example, Brandon used the game to play a female character and express characteristics and behaviour traditionally interpreted as being "feminine," which he states are a part of his upbringing, his interests and who he is, but not conducive to how he wishes to present himself as a man. This echoes Truong's (2013) discussion of how "doing gender" in role-playing practices allows one to play with social norms and social categories, almost inevitably reiterating them while also challenging them in a fluid process.

Finally, TTRPGs had a therapeutic effect in participants' lives by helping participants develop social skills they then mobilized in and out of play. We know that role-playing is found in more traditional forms of therapy, such as cognitive behavioural therapy, which engages role-play in order to rehearse new lifestyles, learn to identify and change thinking 
processes, and gain new social skills (Milkman \& Wanburgh, 2017). In TTRPGs, this process naturally comes about as players adopt a character and invest their own feelings and experiences in the game and the interactions. For example, Adam, who described having trouble socializing with other children and who also experienced bullying, explained that this structured play time helped him to "figure out" social situations in a way that felt safe. Other participants also stated that TTRPGs offered them an opportunity to practice new ways of being which helped them develop a variety of skills. For example, James describes how he used the game to develop his style of humour through role-playing. Participants also played characters who were more outgoing and socially confident, helping them develop stronger interpersonal skills.

Participation in TTRPGs also helped participants develop self-expression skills and a sense of self-advocacy, which can be defined as "the act of supporting one's own interests" (Doherty et al., 2016, p. 253). Conducive to this, leisurebased intervention programmes are believed to allow the individual to take control of their own intervention (Rowe, 2014). Through playing TTRPGs, participants described learning to be "more assertive," to have "more tolerance" for people not liking what they said, and to assert their own independence. This was facilitated through the support, recognition, and validation of other players in the group. For example, Ashley states:

Through D\&D, I learned a lot more of like, I learned a lot more how to be in charge of things, how to have a lot more tolerance for people not liking what I said, how to be a lot truer to myself instead of being customer service [Ashley]. And people still liked it, and people still found me funny, so, I got a lot better at almost every aspect of interpersonal stuff over the time that I've been playing.

Here, Ashley makes a concrete link between her experiences of play and her experiences of life outside of play, and how things she has learned through play become relevant outside that specific context. Other examples include Suzan, creating a transgender advocacy and support group, something she felt empowered to do through her participation in TTRPGs, or Andrew, describing how TTRPGs "changed his life":

I would like to say that they've impacted me for better [...] it made me broaden my horizons so to speak [...] uhm, it made me take dives or like risks. I mean taking chances and all that [...] Umm, like I think the very first few times I've actually played with other people were the first times I've ever made independent decisions for myself. [...] And so, and so, if like, and this is like one of my first few steps to obtaining independence [...] But personally I think it's just one of those stepping stones that I took for me to become who I am today. If I never had that chance back in the day, I wouldn't know what would happen to me.

We know that leisure-based interventions help individuals develop useful skills and self-esteem as well as integrate them into the community (Ponce \& Rowe, 2018). Tabletop roleplaying games can similarly help participants develop these skills as well as form social ties through their participation in gaming groups, local game shops, conventions, or online discussions. However, the role-playing aspect of these games, mixed with their openness to players' experiences and desires, is a rare feature-one that enables a sort of connection with "what is played" that makes it more than fiction.

\section{CONCLUSION}

How do TTRPGs help prevent experiences of social exclusion in youths and young adults? These practices allowed participants to explore and discover different elements of themselves, and the world around them, as well as opportunities to express themselves. The therapeutic qualities of these practices went beyond simply discussing and exploring issues; they were an opportunity to gain new skills and confidence-which were fundamental to improving the participants' lives, well-being, and experiences outside of the game. Furthermore, participants shared that they had developed self-expression skills and a sense of self-advocacy, which encouraged them to support their own interests and build healthy relationships.

We know that encouraging participation of young adults in mental health-related services and keeping them involved can bring many challenges (Truong et al., 2019). We believe that such community organizations could invest in cultural practices that make sense for and are of interest to these individuals. While the sample size of this study may have been limited and precludes generalization, it opens a reflection on the potential therapeutic benefits of these types of practices. We believe more targeted research on the therapeutic potential of TTRPGs is required, as well as on other similar popular culture-related practices for adolescents and adults.

\section{CONFLICT OF INTEREST DISCLOSURES}

The authors declare that there are no conflicts of interest.

\section{AUTHOR AFFILIATIONS}

* Department of Criminology, Faculty of Social Sciences, University of Ottawa, Ottawa, ON, Canada.

\section{REFERENCES}

Barnes, L. (2018, May 25). Dungeons \& dragons: The revival of a "geeky" pastime. BBC News. https://www.bbc.com/news/ukengland-42874044

Binfet, J. (2017). The effects of group-administered canine therapy on university students' wellbeing: A randomized controlled trial. Anthrozoös, 30(3), 397-414.

Blackmon, W. D. (1994). Dungeons and dragons: The use of a fantasy game in the psychotherapeutic treatment of a young adult. American Journal of Psychotherapy, 48(4), 624-632.

Bowman, S. L. (2010). The functions of role-playing games: How participants create community, solve problems and explore identity. McFarland \& Company.

Brooker, D., \& Duce, L. (2000). Wellbeing and activity in dementia: A comparison of group reminiscence therapy, structured goal-directed group activity and unstructured time. Aging \& Mental Health, 4(4), 354-358 
Caltabiano, M. L. (1995). Main and stress-moderating health benefits of leisure. Loisir et Société / Society and Leisure, 18(1), 33-51.

CBS. (September 15, 1985). Special on Dungeons and Dragons. [Television series episode]. In 60 Minutes. CBS Broadcasting. https://archive.org/details/60_minutes_on_dungeons_and_ dragons/part_2.flv

Chen, C., \& Chippendale, T. (2018). Leisure as an end, not just a means, in occupational therapy intervention. The American Journal of Occupational Therapy, 72(4). https://doi.org/10.5014/ ajot.2018.028316.

Cohen, N. (2014, September 13). We're all nerds now. The New York Times. https://www.nytimes.com/2014/09/14/sunday-review/ were-all-nerds-now.html

Coyne, S., Gundersen, N., Nelson, D., \& Stockdale, L. (2011). Adolescents' prosocial responses to ostracism: An experimental study. The Journal of Social Psychology, 151(5), 657-661.

Dempsey, M., Dempsey, P., \& Pulling, P. (n.d.). Dungeons and dragons. http://www.theescapist.com/BADDbook.htm

Doherty, C., Landry, H., Pate, B., \& Reid, H. (2016). Impact of communication competency training on nursing students' selfadvocacy skills. Nurse educator, 47 (5), 252-255.

Frigon, S. (2010). La danse en prison, une échappée belle hors des murs? Perspectives des artistes et des détenues. Criminologie, 43(2), 179-197.

Gaudet, S., \& Robert, D. (2018). A journey through qualitative research: From design to reporting. SAGE Publications Ltd.

Heard, E. M., Mutch, A., Fitzgerald, L., \& Pensalfini, R. (2013). Shakespeare in prison: Affecting health and wellbeing. International Journal of Prisoner Health, 9(3), 111-123.

Jahromi, N. (2017, October 24). The uncanny resurrection of dungeons \& dragons. The New Yorker. https://www.newyorker.com/culture/ cultural-comment/the-uncanny-resurrection-of-dungeons-and-dragons

Kraus, S. (2018, June 27). Edmonton kids learning through fantastical story-telling game. Global News. https://globalnews.ca/ news/4299064/edmonton-students-dungeons-and-dragons-game/

Kushner, D. (2017). Rise of the dungeon master: Gary Gygax and the creation of D\&D. Hachette Book Group USA Perseus.

Lynn Mulvey, K., Boswell, C., \& Zheng, J. (2017). Causes and consequences of social exclusion and peer rejection among children and adolescents. Report on Emotional \& Behavioral Disorders in Youth, 17/3), 71-75.

Martin, D. D., \& Fine, G. A. (1991). Satanic cults, satanic play: Is dungeons \& dragons a breeding ground for the devil? In J. Richardson, D.
Bromley, \&J. Best (Eds.), The Satanism scare (pp. 107-123). Aldine De Gruyter.

Milkman, H., \& Wanburgh, K. (2017). Cognitive-behavioral treatment: A review and discussion for corrections professionals (Rep.). https:// s3.amazonaws.com/static.nicic.gov/Library/021657.pdf

Ponce, A., \& Rowe, M. (2018). Citizenship and community mental health care. American Journal of Community Psychology, 61 (1-2), 22-31.

Rosselet, J. G., \& Stauffer, S. D. (2013). Using group role-playing games with gifted children and adolescents: A psychosocial intervention model. International Journal of Play Therapy, 22(4), 173-192.

Rowe, M. (2014). Citizenship and mental health. Oxford University Press.

Rudnick, A. (2005). Psychiatric leisure rehabilitation: Conceptualization and illustration. Psychiatric Rehabilitation Journal, 29(1), 63-65.

Schiesel, S. (2008, March 5). Gary Gygax, game pioneer, dies at 69. The New York Times. https://www.nytimes.com/2008/03/05/ arts/05gygax.html

Smallfield, S., \& Molitor, W. (2018). Occupational therapy interventions supporting social participation and leisure engagement for community-dwelling older adults: A systematic review. The American Journal of Occupational Therapy, 72(4).

Stanley, L., \& Arora, T. (1998). Social exclusion amongst adolescent girls: Their self-esteem and coping strategies. Educational Psychology in Practice, 14(2), 94-100.

Sweeney, S. (2009). Art therapy: Promoting wellbeing in rural and remote communities. Australasian Psychiatry: Bulletin of the Royal Australian and New Zealand College of Psychiatrists, 17/s1), S151-S154.

Truong, A., \& Gaudet, S. (2020). Costume play and young adults' socioeconomic insertion pathways in Japan. International Journal of the Sociology of Leisure, 3, 177-196.

Truong, A., Larose-Hébert, K., \& Nault, G. (2019). Psychiatrisation et transition à l'âge adulte: La perspective des intervenant-e-s. Aporia, 17(1). https://doi.org/10.18192/aporia.v1 1i1.4490

Truong, A. (2013). Framing cosplay: How 'layers' negotiate body and subjective experience through play. Intersections: Gender and Sexuality in Asia and the Pacific, 32. http://intersections.anu.edu. au/issue $32 /$ truong.htm

United Nations. (2016). Leaving no one behind: The imperative of inclusive development. Report on the World Social Situation 2016. https:// www.un.org/esa/socdev/rwss/2016/full-report.pdf

Wyatt, J., Schwalb, R. J., \& Cordell, B. R. (2014). Dungeons \& dragons player's handbook. Wizards of the Coast.

Zayas, L. H., \& Lewis, B. H. (1986) Fantasy role-playing for mutual aid in children's groups. Social Work with Groups, 9(1), 53-66. 